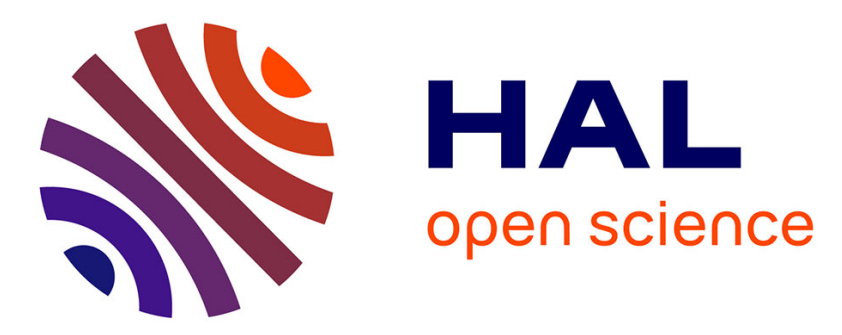

\title{
An extended and integrated digital image correlation technique applied to the analysis of fractured samples
}

Julien Réthoré, Stéphane Roux, François Hild

\section{To cite this version:}

Julien Réthoré, Stéphane Roux, François Hild. An extended and integrated digital image correlation technique applied to the analysis of fractured samples: The equilibrium gap method as a mechanical filter. Revue Européenne de Mécanique Numérique/European Journal of Computational Mechanics, 2009, 18, pp.285-306. 10.3166/ejcm.18.285-306 . hal-00200643

\section{HAL Id: hal-00200643 \\ https://hal.science/hal-00200643}

Submitted on 11 Jan 2008

HAL is a multi-disciplinary open access archive for the deposit and dissemination of scientific research documents, whether they are published or not. The documents may come from teaching and research institutions in France or abroad, or from public or private research centers.
L'archive ouverte pluridisciplinaire HAL, est destinée au dépôt et à la diffusion de documents scientifiques de niveau recherche, publiés ou non, émanant des établissements d'enseignement et de recherche français ou étrangers, des laboratoires publics ou privés.

\section{다)(1) $(5$}

Distributed under a Creative Commons Attribution - NonCommerciall 4.0 International 


\title{
An extended and integrated digital image correlation technique applied to the analysis of fractured samples
}

\section{The equilibrium gap method as a mechanical filter}

\author{
Julien Réthoré — Stéphane Roux — François Hild \\ LMT-Cachan (ENS Cachan/CNRS/Université Paris 6/UniverSud Paris) \\ 61 av. du Président Wilson, F-94230 Cachan, France \\ \{julien.rethore,stephane.roux,francois.hild\}@lmt.ens-cachan.fr
}

\begin{abstract}
RÉSUMÉ. Afin de réduire l'incertitude de mesure, une technique de mesure de champ de déplacement est proposée. Elle associe à la corrélation d'images numériques une pénalisation supplémentaire sur l'écart du champ de déplacement à sa projection dans l'espace des solutions élastiques. Cette application s'effectue dans le cadre de la méthode des élément finis étendus qui permet d'introduire des discontinuités indépendamment du maillage. Une application à l'analyse de la rupture fragile d'une éprouvette de carbure de silicium sert d'illustration à la méthode. Pour compléter l'analyse, la détermination de la position de la pointe de la fissure et des facteurs d'intensités des contraintes est proposée. Ainsi, la chaîne d'acquisition et d'identification est entièrement caractérisée en termes d'incertitude de mesure.

ABSTRACT. To reduce the measurement uncertainty, a measurement technique is proposed for estimating full displacement fields by complementing digital image correlation with an additional penalization on the distance between the estimated displacement field and its projection onto the space of elastic solutions. The extended finite element method is used for inserting discontinuities independently of the underlying mesh. An application to the brittle fracture of a silicon carbide specimen is used to illustrate the application. To complete the analysis, the crack tip location and the stress intensity factors are estimated. This allows for a characterization of the measurement and identification procedure in terms of uncertainty.
\end{abstract}

MOTS-CLÉS: corrélation d'images numériques, méthode de l'écart à l'équilibre, méthode des élément finis étendus

KEYWORDS: digital image correlation, equilibrium gap method, extended finite element method 


\section{Introduction}

Full displacement field measurements by digital image correlation are now widely used in mechanical engineering and materials science (Sutton et al., 2000). One of the main interest is that digital image correlation yields a considerable amount of data that are useful for identification purposes. Fracture mechanics has already been investigated using displacement field measurements (McNeill et al., 1987; AnbantoBueno et al., 2002; Forquin et al., 2004; Réthoré et al., 2005; Roux et al., 2006). The fact that the displacement measurements achieve uncertainties of the order of $10^{-2}$ pixel, allow this technique to reveal cracks that cannot be seen by other means if their opening is less than one pixel wide. Besides the qualitative aspect of detecting a crack, the next challenge is to be able to reach a quantitative estimate of mechanically significant properties, such as stress intensity factors. One related key quantity is the precise determination of the crack tip location, which has a direct consequence on the amplitude of stress intensity factors. This is the problem that is addressed in the present experimental case study, although the technique has a much broader scope of application.

In order to proceed, the first step consists in using a digital image correlation tool that accounts accurately for the kinematics of a cracked specimen. Recently, Réthoré et al. (2007) proposed an extended digital image correlation (X-DIC) technique. It is referred to as "extended" because of the enriched kinematics it uses as in extended finite elements (Moës et al., 1999). A discontinuous enrichment is added to a standard finite element approximation $\left(N_{n}\right)_{n \in \mathcal{N}}$ exploiting its partition of unity property

$$
\sum_{n \in \mathcal{N}} N_{n}(\mathbf{x})=1
$$

The enriched approximation of the displacement field then reads

$$
\mathbf{u}(\mathbf{x})=\sum_{j=1,2} \sum_{n \in \mathcal{N}} a_{n j} N_{n}(\mathbf{x}) \mathbf{X}_{j}+\sum_{j=1,2} \sum_{n \in \mathcal{N}_{c u t}} d_{n j} N_{n}(\mathbf{x}) H_{n}(\mathbf{x}) \mathbf{X}_{j}
$$

where $\left(a_{n j}\right)_{n \in \mathcal{N}}$ are degrees of freedom associated with standard (i.e., continuous) shape functions in each direction $\mathbf{X}_{1}$ and $\mathbf{X}_{2}$ of the image co-ordinate system, $\mathcal{N}_{\text {cut }}$ the set of nodes that hold additional degrees of freedom $d_{n j}$ associated with the enrichment function $H_{n}(\mathbf{x})$ defined as

$$
H_{n}(\mathbf{x})=\mathcal{H}(\mathbf{x})-\mathcal{H}\left(\mathbf{x}_{n}\right)
$$

where $\mathcal{H}$ is the Heaviside step function whose value is 0 below the crack front and 1 above (Figure 1), and $\mathbf{x}_{n}$ gives the position of node $n$. This enrichment function, first introduced by $\mathrm{Zi}$ et al. (2003), is preferred to the Heaviside or to the generalized Heaviside function.

In experimental applications, the advantages of adding enriched degrees of freedom are useful since they allow one to account more precisely for features such as 


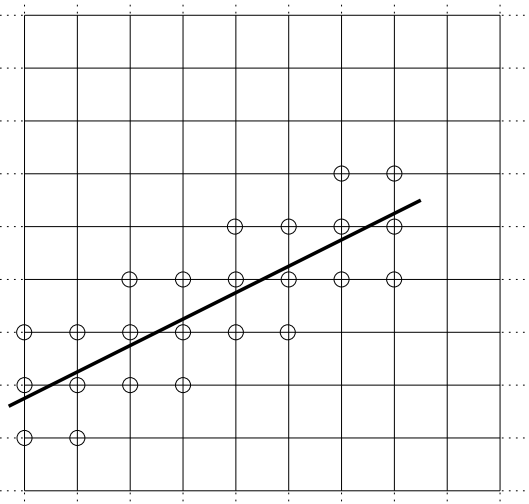

Figure 1. Typical enrichment configuration. Circles denote nodes with discontinuous additional degrees of freedom.

weak or strong discontinuities (e.g., cracks, shear bands) that may be poorly captured by standard finite element shape functions. However, their determination is typically based on the exploitation of pixel values that are less numerous than for standard elements. As a result, the conditioning of the linear system to solve relative to the enriched degrees of freedom is worse than for standard degrees of freedom, and hence they are much more prone to noise. This effect may be compensated for at the identification stage by improving the noise robustness of the stress intensity factor estimation (Réthoré et al., 2006).

In this paper, we propose a different route called extended and "integrated" digital image correlation (or XI-DIC). "Integrated" refers to the fact that the assumed material behavior and the balance of momentum are directly integrated into the correlation algorithm. This type of approach was already used when a closed-form solution exists for Brazilian tests or cracks (Hild et al., 2006; Roux et al., 2006). In the present case, an X-FEM simulation will give access to a mechanically admissible solution. The principle is to perform image correlation with the addition of mechanical constraints so that the distance of the displacement field to the space of mechanically admissible solutions is penalized. A mechanically admissible solution is such that the stress field obtained through a linear stress/strain relationship is momentum balanced. Under these constraints, lower uncertainty levels are expected for the determination of the measured displacement field, and the stress intensity factors.

It is proposed to use the Equilibrium Gap Method coupled with an image correlation algorithm over the entire region of interest. The Equilibrium Gap Method was first used to identify material (e.g., elastic) property fields from a previously measured displacement field (Claire et al., 2004). This identification technique is based on a minimization of the norm of the internal force residuals induced by material parame- 
ter mismatch. In the present paper, it is assumed that the material behavior is linear elastic and the Equilibrium Gap Method is used to enforce the mechanical admissibility of the solution. Both aspects of the method, namely, image correlation and mechanical filtering, are presented in Section 2.

Furthermore, when using an enriched interpolation, the crack geometry has to be determined in advance. As illustrated in Section 4, a mere approximation of the crack path may be obtained from a standard digital image correlation result. One captures the crack faces that hold the discontinuity of the displacement. Even though a good approximation of the crack direction is obtained by visual inspection, the positioning of the crack tip is a sensitive issue (Roux et al., 2006). Moreover, the studied case is expected to be very difficult because the maximum displacement discontinuity is very small, by far sub-pixel (with a value of about 0.3 pixel). Therefore, at a distance of about 15 pixels from the crack tip, the opening is expected to be of the order of $2.5 \times 10^{-2}$ pixel, and hence it is extremely difficult to locate the crack tip very accurately.

When carrying out a correlation analysis, one may have the crack traversing the entire image because, if no discontinuity occurs in the displacement field then the enriched degrees of freedom will be estimated to 0 (to the measurement uncertainty). That is, "optical" tractions make the enriched degrees of freedom vanish over the uncracked region of the image. Accounting for balance of momentum in the correlation algorithm, the crack faces are traction-free from a mechanical point of view and the activation of all the enriched degrees of freedom is unavoidable. Consequently, an integrated approach needs a more accurate positioning of the crack tip. Thus, Section 5 is aimed at presenting how the integrated approach is used to determine the crack tip position. Last, stress intensity factors are estimated with an interaction integral.

\section{Integrated digital image correlation}

In the following, an integrated correlation algorithm is presented. It is based upon a correlation procedure that is regularized by seeking mechanically admissible displacement fields.

\subsection{Image correlation}

The correlation of two gray level images $f$ and $g$ ( $f$ is the reference picture and $g$ the deformed one) is recast as the local passive advection of the texture of the two images by a displacement field $\mathbf{u}$

$$
g(\mathbf{x})=f(\mathbf{x}+\mathbf{u}(\mathbf{x}))
$$

The problem consists in finding the best displacement field by minimizing the correlation residual

$$
\eta(\mathbf{x})=|f(\mathbf{x}+\mathbf{u}(\mathbf{x}))-g(\mathbf{x})|
$$


The minimization of $\eta$ is intrinsically a non-linear and ill-posed problem. For these reasons, a discrete and weak format is preferred by adopting a general discretization scheme

$$
\mathbf{u}(\mathbf{x})=\sum_{n \in \mathcal{N}} a_{n} \boldsymbol{\psi}_{n}(\mathbf{x})=[\mathbf{\Psi}(\mathbf{x})]\{\mathbf{U}\}
$$

where $\psi_{n}$ are the vector shape functions (including the enhanced ones) and $a_{n}$ their associated degrees of freedom. In a matrix-vector format, $[\Psi]$ is a row vector containing the values of the shape functions $\boldsymbol{\psi}_{n}$ and $\{\mathbf{U}\}$ the column vector of the degrees of freedom. After integration over the domain $\Omega$, the global residual is defined as

$$
\mathrm{R}_{\text {cor }}=\iint_{\Omega}(f(\mathbf{x}+[\mathbf{\Psi}(\mathbf{x})]\{\mathbf{U}\})-g(\mathbf{x}))^{2} \mathrm{~d} \mathbf{x}
$$

At this level of generality, many choices can be made to measure displacement fields. In the following, an extended procedure is considered (Réthoré et al., 2007), based upon a Q4 correlation algorithm (Besnard et al., 2006).

\subsection{Balance of momentum}

To enforce mechanical admissibility in a FE sense, the following mechanical residual is introduced. The local balance of momentum equation reads

$$
\nabla \cdot \sigma=\mathbf{0}
$$

and is reformulated as an "energy" term based on the domain integral of the quadratic norm of the above divergence, in a form suitable to the used finite element formulation. Let us first recall the expression of the strain energy

$$
\mathrm{E}_{m e c}=(1 / 2) \iint_{\Omega} \boldsymbol{\sigma}: \boldsymbol{\epsilon} \mathrm{d} \mathbf{x}
$$

where ":" denotes the contraction with respect to two indices, $\sigma$ the Cauchy stress tensor and $\varepsilon$ the infinitesimal strain tensor. Prescribing a linear elastic stress/strain relationship $\sigma=\mathcal{C}: \varepsilon$, where $\mathcal{C}$ is the fourth order elastic tensor, and adopting the discretization scheme for the displacement, the matrix-vector format of $\mathrm{E}_{m e c}$ is

$$
\mathrm{E}_{m e c}=(1 / 2)\{\mathbf{U}\}^{T}[\mathbf{K}]\{\mathbf{U}\}
$$

where $\mathbf{K}$ is the stiffness matrix associated with the discretization scheme and $\{\mathbf{U}\}$ contains the values of the displacement unknowns. If $\nabla^{s}$ denotes the symmetrized gradient in space, then the components of $\mathbf{K}$ read

$$
\mathrm{K}_{n m}=\iint_{\Omega} \nabla^{s} \boldsymbol{\psi}_{m}: \mathcal{C}: \nabla^{s} \boldsymbol{\psi}_{n} \mathrm{~d} \mathbf{x}
$$


For identification purposes, the minimization of the mechanical energy is more conveniently written as the norm of the internal forces in the spirit of the Equilibrium Gap Method (Claire et al., 2004). Then, $\mathrm{R}_{m e c}$ is defined as

$$
\mathrm{R}_{m e c}=(1 / 2)\{\mathbf{U}\}^{T}[\overline{\mathbf{K}}]^{\mathrm{T}}[\overline{\mathbf{K}}]\{\mathbf{U}\}
$$

where $[\overline{\mathbf{K}}]$ is the rectangular part of $[\mathbf{K}]$ involved in the computation of the internal forces of the internal nodes of the mesh (i.e., all nodes except those on the mesh boundary).

\subsection{Partitioning the residuals}

To solve the coupled minimization of image correlation residual $\mathrm{R}_{\text {cor }}$ and mechanical admissibility residual $\left(\mathrm{R}_{m e c}\right)$, a weighted sum of both residuals to a total potential $\mathrm{R}_{\text {tot }}$ is defined as

$$
\mathrm{R}_{\text {tot }}=(1-\lambda) \mathrm{R}_{\text {mec }}+\lambda \mathrm{R}_{\text {cor }}
$$

where $\lambda$ is a coupling parameter belonging to $[0 ; 1]$. In practice, $\mathrm{R}_{m e c}$ and $\mathrm{R}_{c o r}$ are normalized by their initial value when $\lambda$ is unity, namely $\mathrm{R}_{m e c}^{0}$ and $\mathrm{R}_{c o r}^{0}\left(\mathrm{R}_{\text {tot }}^{0}=\right.$ $\mathrm{R}_{c o r}^{0}$ ) i.e., for a displacement field obtained by a standard (e.g., Q4-DIC) correlation technique as an initialization step of the analysis.

\section{Resolution}

For a given value of $\lambda$, the minimization of $\mathrm{R}_{t o t}$ with respect to $\{\mathbf{U}\}$ is nonlinear because of the presence of $\mathrm{R}_{\text {cor }}$. A Newton iterative procedure is elaborated to circumvent this non-linear aspect of the problem. Let $\mathbf{U}^{i}$ denote the displacement at iteration $i$. Then assuming small increments $\mathrm{d} \mathbf{U}=\mathbf{U}^{i+1}-\mathbf{U}^{i}$ of the solution, the minimization of $\mathrm{R}_{t o t}$ reads

$$
\left\{\frac{\partial \mathrm{R}_{t o t}}{\partial \mathbf{U}}\right\}_{\lambda}^{i}=(1-\lambda)[\tilde{\mathbf{K}}]\{\mathbf{U}\}+\lambda\left\{\frac{\mathrm{dR}_{\text {cor }}}{\mathrm{d} \mathbf{U}}\right\}^{i}
$$

with

$$
[\tilde{\mathbf{K}}]=[\overline{\mathbf{K}}]^{T}[\overline{\mathbf{K}}]
$$

Under the same assumption of small increment of the solution, a Taylor expansion is used to linearize $f(\mathbf{x}+\mathbf{u}(\mathbf{x}))$ and $\left\{\frac{\mathrm{dR}_{\text {cor }}}{\mathrm{d} \mathbf{U}}\right\}^{i}$ is recast in a matrix-vector product

$$
\left\{\frac{\mathrm{dR}_{\text {cor }}}{\mathrm{d} \mathbf{U}}\right\}^{i}=[\mathbf{M}]^{i}\{\mathrm{~d} \mathbf{U}\}-\{\mathbf{b}\}^{i}
$$

with

$$
M_{n m}^{i}=\iint_{\Omega}\left(\boldsymbol{\psi}_{n} \cdot \nabla f\left(\mathbf{x}+[\Psi]\{\mathbf{U}\}^{i}\right)\right)\left(\boldsymbol{\psi}_{m} \cdot \nabla f\left(\mathbf{x}+[\Psi]\{\mathbf{U}\}^{i}\right)\right) \mathrm{d} \mathbf{x}
$$


and

$$
b_{n}^{i}=\iint_{\Omega}\left(\boldsymbol{\psi}_{n} \cdot \nabla f\left(\mathbf{x}+[\Psi]\{\mathbf{U}\}^{i}\right)\right)\left(g(\mathbf{x})-f\left(\mathbf{x}+[\Psi]\{\mathbf{U}\}^{i}\right)\right) \mathrm{d} \mathbf{x}
$$

A key point in reaching good convergence rates is the computation of $\nabla f\left(\mathbf{x}+\mathbf{u}^{i}\right)$. The gradient of the image $f$ is computed in the frequency space using an FFT algorithm. For consistency, FFT is also used to translate the image for the sub-pixel parts of $\mathbf{u}^{i}$.

The increment of the solution is thus obtained by solving

$$
\left[(1-\lambda)[\tilde{\mathbf{K}}]+\lambda[\mathbf{M}]^{i}\right]\{\mathrm{d} \mathbf{U}\}=\lambda\{\mathbf{b}\}^{i}-(1-\lambda)[\tilde{\mathbf{K}}]\{\mathbf{U}\}^{i}
$$

The solution is then updated and the process is repeated until a convergence criterion associated with a given norm $\left|\mathrm{R}_{\text {tot }}^{0}-\mathrm{R}_{\text {tot }}^{i}\right| /\left|\mathrm{R}_{\text {tot }}^{0}\right|$ is reached.

One key question is the determination of the $\lambda$ value to be chosen (at present this parameter appears as arbitrary):

- One option is to impose that the displacement field be admissible. This corresponds to considering the limit $\lambda \rightarrow 0^{+}$. It may be shown that this limit corresponds to imposing the displacement field measured on the outer boundary of the domain, and to solve for the Dirichlet elastic problem with this boundary condition to obtain the displacement in the bulk of the domain. This approach is quite satisfactory, with however one limitation. The outer boundary is where the determination of the displacement has the least confidence level, and thus the elastic solution matching the outer boundary is presumably not the most noise-robust determination.

- An alternative corresponds to fixing a non-zero value of $\lambda$. In this case, it may be shown that there exists a length scale, $\xi$, such that

$$
\xi \propto \sqrt[4]{\frac{1-\lambda}{\lambda}}
$$

and the additional mechanical term in the residue is such that over wavelengths less than $\xi$, internal forces are used to relax the displacement field, whereas larger wavelengths are preserved and quenched. Thus, considering a finite $\lambda$ value may be seen as a way to preserve unphysical internal forces after a low-pass frequency filtering. However, if the length scale $\xi$ is large enough, the magnitude of these internal forces may be considerably dampened and still the largest noise error due to the boundary may have a reduced influence on the bulk determination of the displacement.

Therefore, our tentative conclusion at this stage is to favor the $\lambda \rightarrow 0^{+}$limit, as corresponding to a penalization of non mechanically admissible displacement fields, or to allow for a small but non-zero $\lambda$ value, based on a criterion of noise sensitivity. This is the route followed in the sequel. 


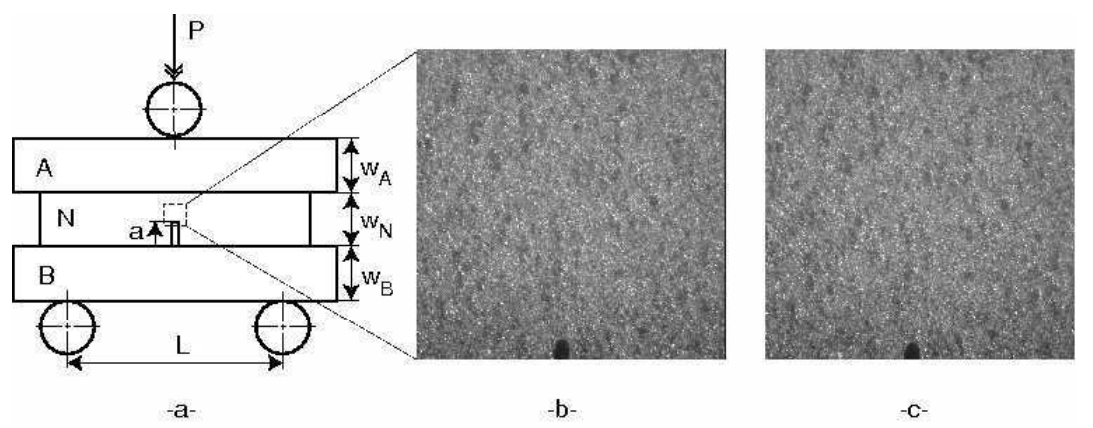

Figure 2. Geometry of the sandwiched beam set-up (a). The brittle sample $(N)$ is put in between two steel beams (A and B). A three-point bend test is performed. A first stable crack is initiated. Initial (b) and cracked (c) SiC sample face. In the middle of the lower side, the black spot is the mark of the notch, from which a crack propagates. The image size is $1008 \times 1016$ pixels with a conversion factor $p=1.85 \mu \mathrm{m} / \mathrm{pixel}$.

\section{Preliminary results}

The application discussed herein is based upon a silicon carbide ( $\mathrm{SiC}$ ) sample in a sandwiched beam test (Figure 2-a). This type of experimental set-up allows one to initiate a crack without complete failure of the central beam. However, the arrest conditions are strongly dependent upon friction between the beams, their flexural stiffness, and the notch geometry (Forquin et al., 2004). Therefore, numerical tools require a number of measurements to feed the modeling before the latter may provide a meaningful determination of stress intensity factors.

The region of interest covers part of the image whose size is $1008 \times 1016$ pixels with an 8-bit digitization (see Figure 3). Preliminary results are obtained with a Q4algorithm. The mesh with 16-pixel elements and the crack geometry are shown in Figure 2-b.

The change of the residuals is presented in Figure 4. It is shown that the equilibrium gap decreases very rapidly from the initial pure correlation solution with $\lambda$ equal to 1 . When the coupling parameter tends to 0 , the mechanical contribution to the energy also vanishes as its weight with respect to the correlation residual becomes larger. Even though $\mathrm{R}_{m e c}$ decreases very rapidly when $\lambda$ is less than $1, \mathrm{R}_{c o r}$ slowly increases (the maximum value of $\mathrm{R}_{c o r}$ is of the same order of magnitude as its initial value) from the residual obtained for a pure correlation problem $(\lambda=1)$. As a consequence, $\mathrm{R}_{c o r}$ remains the dominant term in $\mathrm{R}_{t o t}$ even if its contribution is weighted by $\lambda$. $\mathrm{R}_{t o t}$ is then nearly linear in $\lambda$ and the minimization through the coupling parameter leads to the limit solution with $\lambda$ valued to 0 .

A stabilization of the solution is observed in Figure 5 where the difference between the XI-DIC and X-DIC solutions in terms of mean displacement is plotted as a 

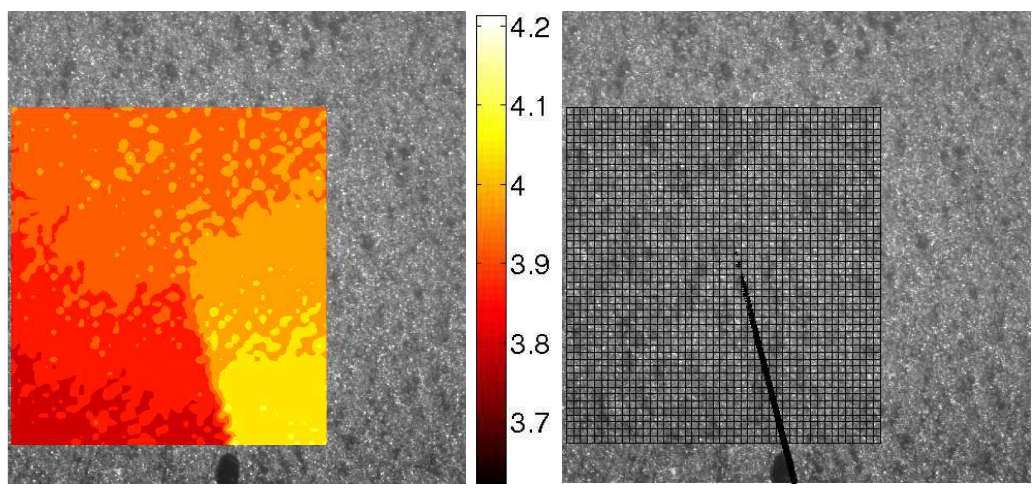

Figure 3. Horizontal displacement map superimposed to the reference image (left) when a Q4-algorithm is used. 16-pixel mesh and initial guess of the crack geometry (right). The conversion factor is $p=1.85 \mu \mathrm{m} /$ pixel.
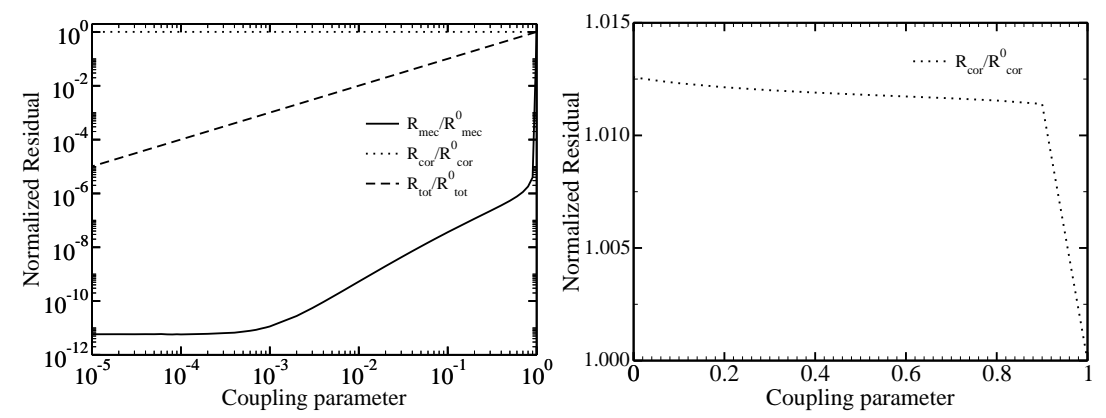

Figure 4. Change of the normalized residuals level as functions of the coupling parameter $\lambda$.

function of the coupling parameter. As mentioned earlier, it is proposed to perform an uncertainty analysis in order to select the appropriate value of the coupling parameter $\lambda$. The reference image is artificially deformed by an asymptotic displacement field that is in the admissible space of searched solutions, namely, the analytical crack solutions in mode I and II are used to create a fake deformed image. The displacement is such that the normal and tangential discontinuities are 0.5 pixel at the bottom of the reference image. This constitutes an a priori test to evaluate the performance in the estimation of the displacement field as compared to known displacements for each value of $\lambda$.

The root mean square (RMS) of the displacement error is plotted in Figure 6. A minimum value, $8.5 \times 10^{-3}$ pixel, of the uncertainty in the displacement jump is obtained for $\lambda=0.25$ although the error does not increase much for smaller values, 


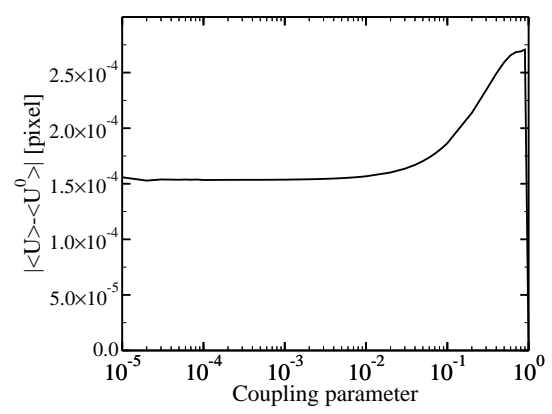

Figure 5. Change of the displacement gap between the XI-DIC and the X-DIC solutions as functions of the coupling parameter $\lambda$.
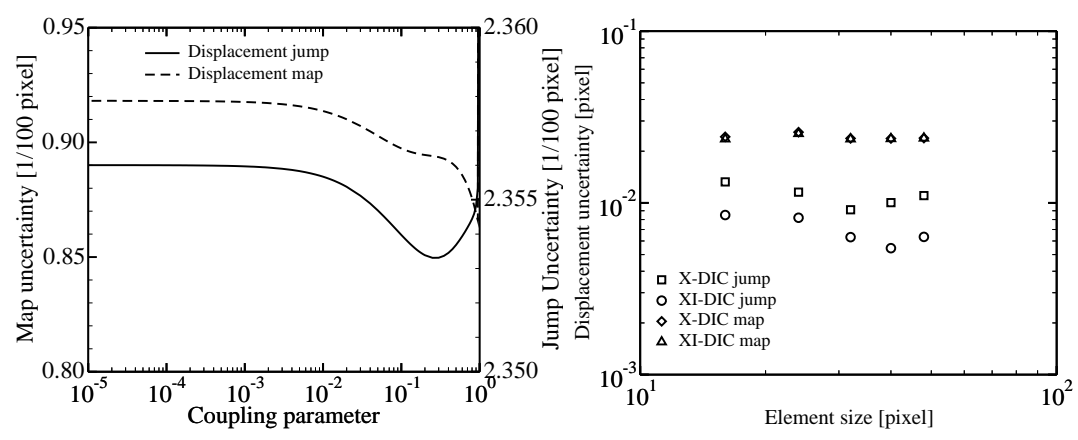

Figure 6. Uncertainty measurement for the displacement jump on an artificially deformed image. Left, uncertainty for 16-pixel elements as a function of coupling parameter; right, uncertainty as a function of element size.

reaching $8.9 \times 10^{-3}$ pixel for $\lambda=10^{-4}$ whereas it was $1.35 \times 10^{-2}$ pixel when $\lambda=1$. The value of $\lambda$ is non-zero but still quite small as compared to 1 , as proposed above. For this optimal choice of $\lambda$, the measurement uncertainty on the displacement map under conditions similar to the experimental analysis (same image texture, same displacement range, same assumed material behavior) is $2.35 \times 10^{-2}$ pixel. Figure 6 also depicts the uncertainty as a function of the element size. One may notice that increasing the element size first has a positive effect (the uncertainty decreases) but then the interpolation error becomes predominant and the uncertainty increases. The gain obtained with the proposed penalization mainly concerns the displacement jump along the crack front. For the latter, enforcing a zero-traction condition enables for a significant decrease of the displacement uncertainty.

Let us now report on the analysis of the pair of experimental images, using first a simple X-DIC algorithm, and second the XI-DIC method with the optimal value of 

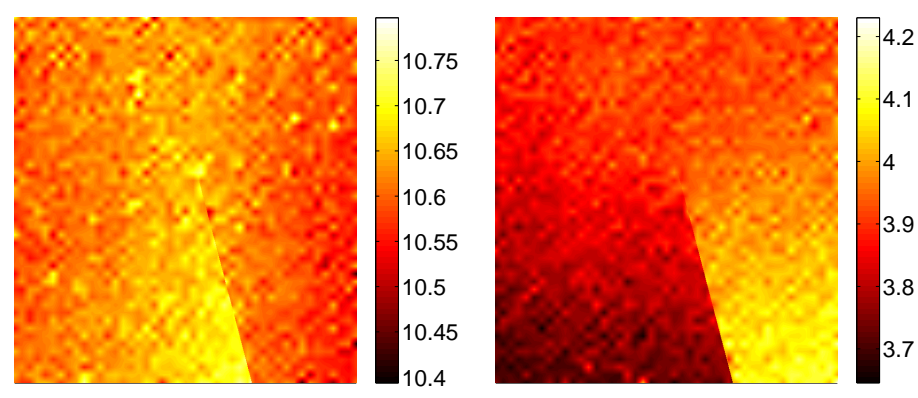

$-a-$
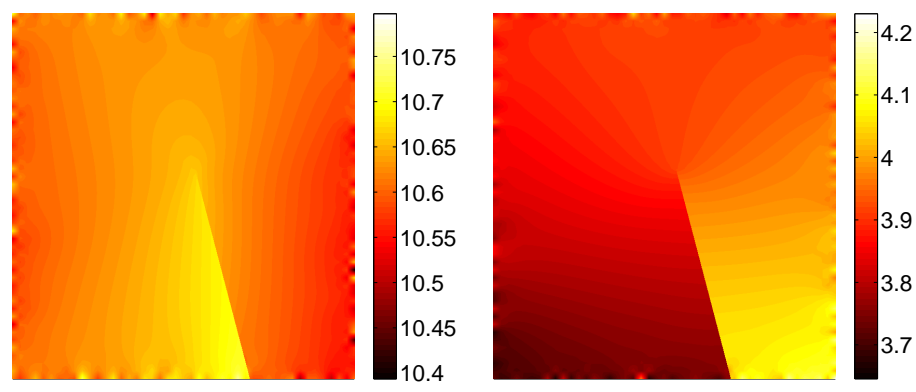

$-b-$

Figure 7. Vertical (left) and horizontal (right) displacement map for the SiC specimen for 16-pixel elements using: X-DIC (a), XI-DIC (b). The conversion factor is $p=$ $1.85 \mu \mathrm{m} /$ pixel.

$\lambda=0.25$ as above determined. The displacement maps are presented in Figure 7. The fields remain similar but the integrated solution shows a noiseless aspect. This corresponds to the expected effect, namely, the short wavelength displacement noise is (efficiently) filtered out by the mechanical penalization term.

However, the two solutions reveal indistinguishable error maps (see Figure 8). The mean values are respectively 3.3 and 3.5 gray levels for the X-DIC and XI-DIC solutions. The incorporation of the admissibility condition restricts considerably the space of displacement fields, and thus it leads to an increase in the optical residual. Yet this increase is very limited, and well within the expected noise level in the image acquisition.

Furthermore, the norm of the difference between the two solutions normalized by the norm of the X-DIC solution is shown in Figure 9. The mean value of the gap is $0.2 \%$ and its maximum $4.6 \%$. This corresponds to the part of the displacement field that was filtered out by the elastic penalization. It is essentially a short range correlated field, likely to be pure noise. 


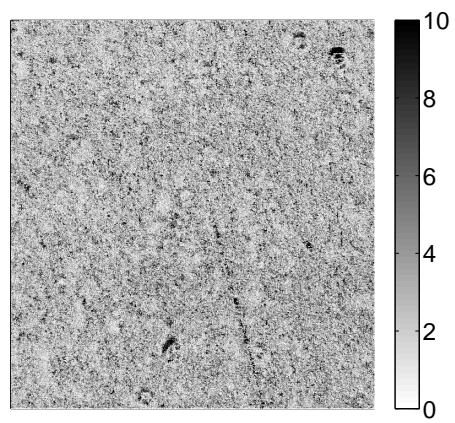

$-a-$

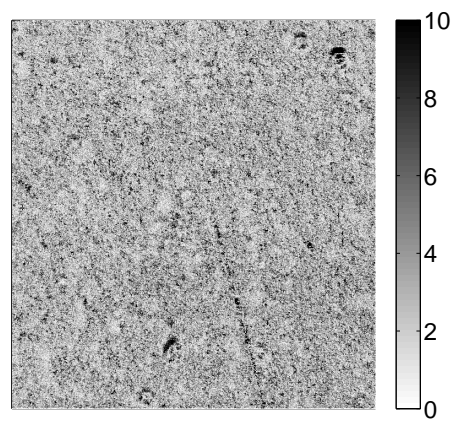

$-b-$

Figure 8. Error map $\eta$ for the SiC specimen for 16-pixels element using X-DIC (a) or XI-DIC (b) algorithms. The analyzed pictures have an 8-bit digitization.

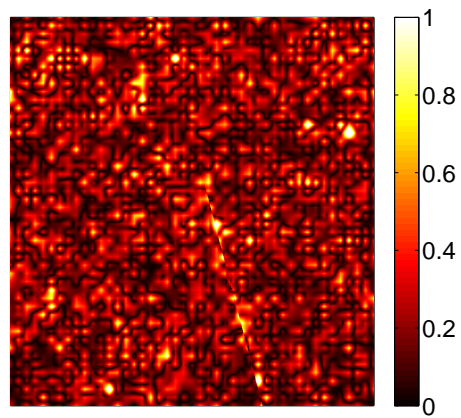

Figure 9. Normalized norm of the gap between the X-DIC and XI-DIC solutions in percents

As mentioned in the introduction of the paper, enriched degrees of freedom associated with jump functions are more sensitive to noise than classical finite element degrees of freedom. Figure 10 shows that the mechanical filtering better constrains the enriched degrees of freedom and the crack opening profile is noiseless except within the boundary element wherein the minimization of the internal forces is not enforced. For X-DIC, the practical displacement uncertainty is less than 0.1 pixel, i.e., less than twice the a priori estimate (Figure 6).

\section{Crack tip positioning}

One key information to be extracted from the displacement analysis of cracked samples is the location of the crack tip. This is difficult since the displacement am- 


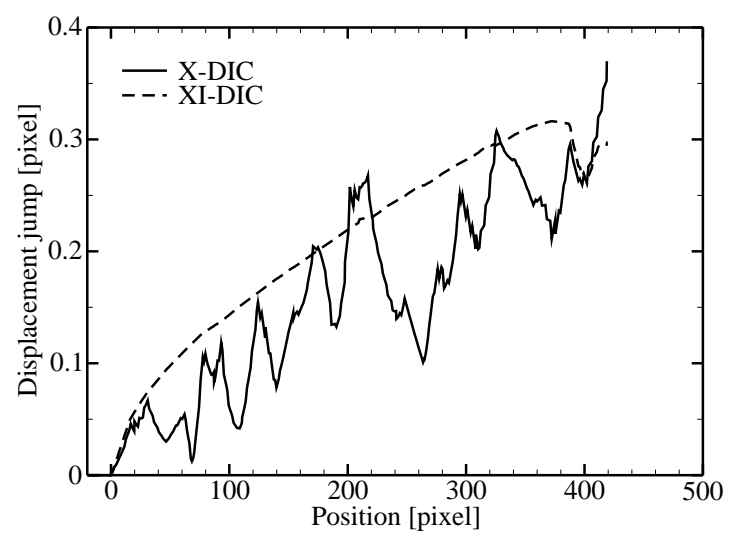

Figure 10. Comparison of the norm of the displacement jump for the X-DIC and the XI-DIC solutions.

plitude in the vicinity of the crack tip are less than the resolution of the measurement technique. We propose a method based on the exploitation of displacement field and of the analytical elastic crack solutions.

\subsection{First super-singular component}

From a theoretical view point, the displacement field solution of a plane elasticity problem with a semi-infinite crack is conveniently written by using KolossovMuskhelishvili potentials (Williams, 1957; Irwin, 1957). The displacement field $\mathbf{u}$ is written in the complex plane $z=x+i y$, as $\mathbf{u}=u_{x}+i u_{y}$. The complete set of linear elastic displacement field satisfying a condition of zero traction along the crack path is written as two integer-labeled families of solutions

$$
\mathbf{u}=\sum_{j, n} a_{n}^{j} \boldsymbol{\Phi}_{n}^{j}
$$

with $j$ indicating the mode (I or II). We only give here the discontinuous (odd $n$ ) solutions. For mode I, the functions $\boldsymbol{\Phi}$ read

$$
\boldsymbol{\Phi}_{n}^{I}=(-1)^{(n+1) / 2} r^{n / 2}\left(\kappa e^{i n \theta / 2}-\frac{n}{2} e^{i(4-n) \theta / 2}+\left(\frac{n}{2}-1\right) e^{-i n \theta / 2}\right)
$$

and for mode II

$$
\boldsymbol{\Phi}_{n}^{I I}=i(-1)^{(n-1) / 2} r^{n / 2}\left(\kappa e^{i n \theta / 2}+\frac{n}{2} e^{i(4-n) \theta / 2}-\left(\frac{n}{2}-1\right) e^{-i n \theta / 2}\right)
$$

where the constant $\kappa$ is equal to $(3-\nu) /(1+\nu)$ in plane stress or $3-4 \nu$ in plane strain, $\nu$ being the Poisson's ratio. The components corresponding to $n=1$ are the 
classical fields giving access to the stress intensity factors. In practice the amplitudes corresponding to this family of solutions are evaluated by using a least-squares minimization of the gap between the DIC displacement and a displacement field including functions for $n=-3$ to $n=3$ for mode $I$ and mode $I I$. Using the above expressions, the following property is derived

$$
\frac{\partial \Phi_{n}^{j}}{\partial x}=-\frac{n}{2} \Phi_{n-2}^{j}
$$

The derivative with respect to the crack tip position $x_{c}$ being the opposite of the above equation, it shows that the mis-positioning of the crack tip along its axis will give rise to a supersingular contribution $\Phi_{-1}^{j}$, away from the immediate vicinity of the crack tip. Therefore, in order to estimate the crack tip position, one may decompose the displacement field using the $n=1$ and $n=-1$ order functions, and rewrite

$$
a_{1}^{j} \Phi_{1}^{j}+a_{-1}^{j} \Phi_{-1}^{j}=a_{1}^{j}\left(\Phi_{1}^{j}+2 \frac{a_{-1}^{j}}{a_{1}^{j}} \frac{\partial \Phi_{1}^{j}}{\partial x_{c}}\right)
$$

This result is interpreted as a Taylor expansion of the usual crack tip field whose tip would be shifted from the actual one by an offset distance $x_{1}$ such that

$$
x_{1}=2 \frac{a_{-1}^{1}}{a_{1}^{1}}
$$

Calculating $x_{1}$ from $a_{-1}^{j}$ and $a_{1}^{j}$, one obtains an estimate of the offset between the assumed crack tip and the actual one. Figure 11a shows $x_{1}$ as a function of the shift of the crack tip. It is observed that a unique position allows one to cancel the first supersingular amplitude, which is defined to be the most likely crack tip position (with respect to its influence on the large distance displacement field). Moreover, Figure 11 shows that the derivative of the offset with respect to the shift has a slope of -1 in the vicinity of the point where the offset vanishes, as expected from the above argument. Thus, from this analysis the best evaluation of the crack tip position is located 43 pixels away from the initially guessed position.

\subsection{Correlation residual}

As an independent check of this result, the correlation residual $\mathrm{R}_{\text {cor }}$ is plotted in Figure $11 \mathrm{~b}$ as a function of the shift of the crack tip in the direction tangent to the crack faces. One observes a minimum of $\mathrm{R}_{c o r}$ for the same shift of about 43 pixels from the a priori assumed position.

\subsection{Crack opening displacement}

Furthermore, Figure 11c also shows the change of the squared displacement jump that is expected to be linear in elasticity. Using this curve, one easily extrapolates 
the far-field results that remain linear to obtain the position of the crack tip where the displacement discontinuity vanishes. A shift of 43 pixels again leads to a very good estimate of the actual position of the crack tip.

Last, Figure 12 allows one to compare the displacement jump obtained by X-DIC and XI-DIC with the initial position of the crack tip and with the crack tip shifted by 43 pixels. Even though the effect of the modification of the crack tip position is significant in the neighborhood of the crack tip, the results remains the same at a distance of about 150 pixels from the shifted tip for XI-DIC.

\section{Stress intensity factors}

\subsection{Interaction integral}

After measuring the displacement field, stress intensity factors are estimated by various techniques, namely, crack opening displacement (Forquin et al., 2004), least squares minimization of the gap between the solution and the theoretical solution (McNeill et al., 1987; Anbanto-Bueno et al., 2002) or interaction integral (Réthoré et al., 2005). Because of the very erratic crack profile obtained with an X-DIC technique (Figure 10), an estimation of stress intensity factors using the crack opening displacement is not appealing. As a domain independent integral, the interaction integral $I^{\text {int }}$ may be more convenient

$$
I^{i n t}=-\int_{D}\left[\sigma_{m l}^{a} u_{m, l} \delta_{k j}-\left(\sigma_{i j}^{a} u_{i, k}+\sigma_{i j} u_{i, k}^{a}\right)\right] q_{k, j} \mathrm{~d} D
$$

where $\mathbf{u}, \sigma$ are the actual displacement and stress fields, $\mathbf{u}^{a}, \sigma^{a}$ the auxiliary displacement and stress fields, $\mathbf{q}$ the virtual crack extension field, and $D$ the domain over which $I^{i n t}$ is computed. The auxiliary field is chosen to be the analytical solution of an infinite elastic body with a semi-infinite crack in mode $I$ or mode $I I$ (i.e., the above introduced $\Phi_{1}^{j}$ fields). Under these assumptions, the stress intensity factors are obtained through the two-field Irwin relationship

$$
I^{i n t}=\frac{2}{E}\left(K_{I} K_{I}^{a}+K_{I I} K_{I I}^{a}\right)
$$

If $K_{I}^{a}=1 \mathrm{MPa} \sqrt{\mathrm{m}}$ and $K_{I I}^{a}=0$, respectively $K_{I}^{a}=0$ and $K_{I I}^{a}=1 \mathrm{MPa} \sqrt{\mathrm{m}}$, then $K_{I}$, respectively $K_{I I}$, are directly evaluated from $I^{i n t}$. The virtual crack extension field has to satisfy the following conditions

$$
\begin{aligned}
\mathbf{q} \cdot \mathbf{n}_{\Gamma}=0 & \text { on } \Gamma \\
\mathbf{q}=\mathbf{t}_{\Gamma} & \text { at the crack tip } \\
\mathbf{q}=\mathbf{0} & \text { on } \partial D
\end{aligned}
$$

where $\Gamma$ is the crack surface and $\mathbf{n}_{\Gamma}, \mathbf{t}_{\Gamma}$ its unit normal and tangent vectors. In the present paper, the norm of $\mathbf{q}$ is assigned to linear variation between $\partial D$ and the crack 
16 Revue européenne des éléments finis. Volume XXXX - no. YYYY/2007

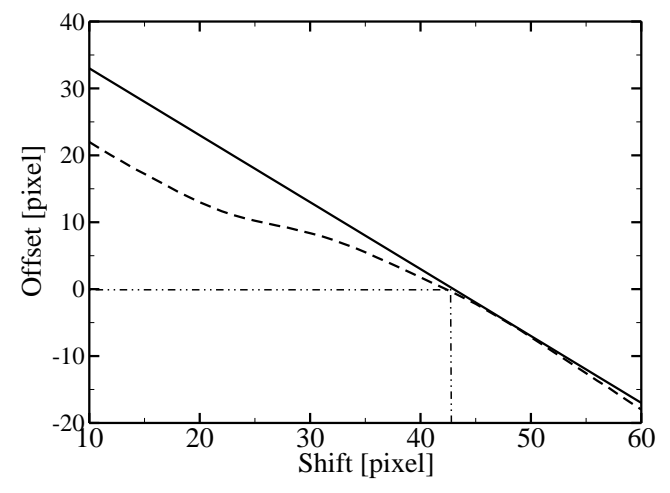

-a-

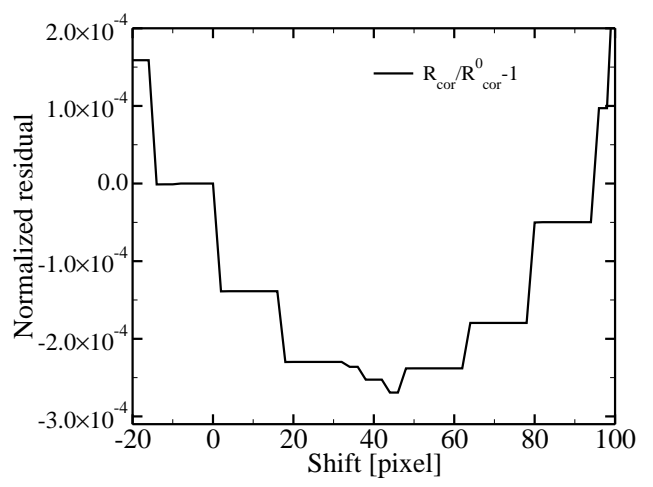

-b-

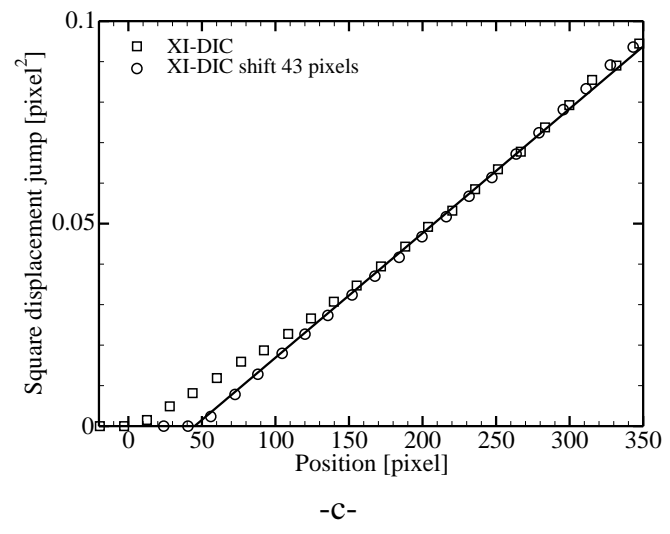

Figure 11. Three methods to determine the crack tip position: (a) estimated offset using the first supersingular term obtained by a least squares fit; (b) change of the normalized correlation residual; (c) square of the norm of the displacement jump obtained by XI-DIC for initial crack tip and the 43-pixel shifted crack tip. 


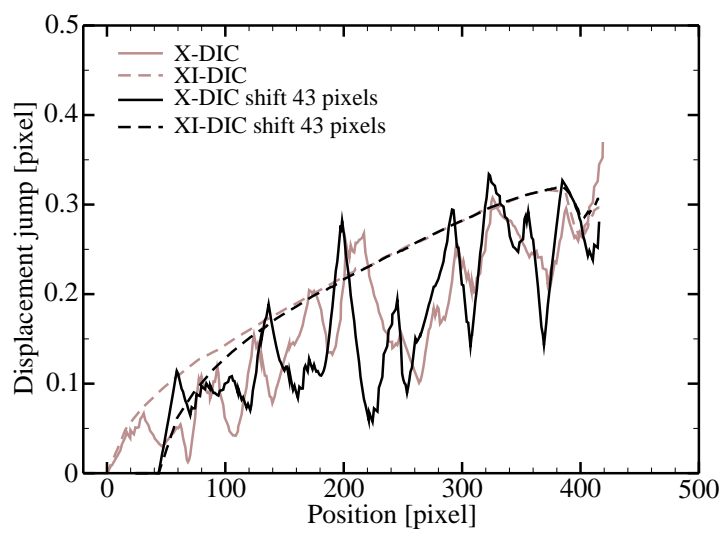

Figure 12. Comparison of the norm of the displacement jump for the X-DIC and XIDIC solutions with the initial crack tip and the shifted crack tip.

tip. If two discretization schemes are adopted for $\mathbf{u}$ and $\mathbf{q}$, then the interaction integral, and thus the stress intensity factors are recast in a matrix-vector format

$$
K_{I, I I}=\{\mathbf{Q}\}^{\mathrm{T}}\left[\mathbf{S}_{I, I I}\right]\{\mathbf{U}\}
$$

where $\{\mathbf{Q}\}$ contains the nodal values of $\mathbf{q}$ evaluated for a standard finite element interpolation over the same mesh as for $\mathbf{u}$. $K_{I, I I}$ et $\mathbf{S}_{I, I I}$ correspond to mode $I$ or mode $I I$ by setting the appropriate auxiliary field in

$$
\mathbf{S}_{I, I I}=-\frac{E}{2} \int_{\Omega} \nabla \psi_{\mathbf{q}}^{\mathrm{T}}\left[\boldsymbol{\sigma}^{a}: \nabla \psi_{\mathbf{u}} \mathbf{I}-\left(\boldsymbol{\sigma}^{a} \nabla \psi_{\mathbf{u}}+\left(\mathbf{D} \nabla^{s} \psi_{\mathbf{u}}\right) \nabla \mathbf{u}^{a}\right)\right] \mathrm{d} \Omega
$$

\subsection{Noise sensitivity}

Let us now assume that the digital images are corrupted by noise during the acquisition process of the camera. This noise is modeled to be white, namely, the noise that affects each pixel of the sensor is uncorrelated with that of neighboring pixels. The image $f$ may be conveniently defined as a noiseless reference, so that the tangent matrix of the correlation residual is not affected by noise. On the other hand, the

residual itself, invoking $g-f$, contains a noise whose variance $\eta_{p}^{2}$ is twice the variance of the actual acquisition noise. From the linear system solved at the last iteration (Equation [18]) of the Newton algorithm, ones obtains the perturbation $\eta_{\boldsymbol{u}}$ induced by this noise on the displacement estimation. Since $f$ is considered to be noiseless, only $\{\mathbf{b}\}^{i}$ is affected by the noise and its variance is recast under the form $[\mathbf{M}]^{i}$. Then, the variance of the displacement perturbation reads

$$
\left\langle\boldsymbol{\eta}_{\boldsymbol{u}}{ }^{2}\right\rangle=\lambda^{2}\left[\left(\mathbf{A}^{i}\right)^{-1} \mathbf{M}^{i}\left(\mathbf{A}^{i}\right)^{-1}\right] \eta_{p}^{2}
$$


where

$$
\left[\mathbf{A}^{i}\right]=\left[(1-\lambda)[\tilde{\mathbf{K}}]+\lambda[\mathbf{M}]^{i}\right]
$$

This is now used to evaluate the perturbation of the estimation of the stress intensity factors. By using Equation [29], the corresponding variance of the stress intensity factors is

$$
\left\langle\delta K_{I, I I}^{2}\right\rangle=\lambda \mathbf{Q}^{\mathrm{T}}\left[\mathbf{S}_{I, I I}\left(\mathbf{A}^{i}\right)^{-1} \mathbf{M}^{i}\left(\mathbf{A}^{i}\right)^{-1} \mathbf{S}_{I, I I}^{T}\right] \eta_{p}^{2}
$$

This last equation defines the perturbation of the stress intensity factors estimation directly expressed as a function of the image noise, and the correlation algorithm. This formula will be used in the sequel to estimate error bars on the determination of the stress intensity factors.

\subsection{Results}

Figure 13a shows the standard deviation of $K_{I}$ as a function of the integration domain size. First, using pure correlation measurements, one may better choose a domain as large as possible. As the domain becomes larger, the gradient of the virtual crack extension that is involved in the interaction integral is decreasing as well as the sensitivity of the estimation. Second, the XI-DIC procedure gives a nearly constant uncertainty if a strong coupling parameter is used $(\lambda=0.0001$ for which the minimum of the mechanical residual is reached, see Figure 4). When the domain size reaches the size of the region of interest, the estimation of the displacement within the outer layer of elements, which is as sensitive as for X-DIC, increases the sensitivity of the estimation of $K_{I}$. However, uncertainties of about $6 \times 10^{-3} \mathrm{MPa} \sqrt{\mathrm{m}} /$ gray level are obtained for the integrated scheme whereas the lower uncertainty obtained with X-DIC was 10 times higher. With $\lambda=0.25$ the change of the uncertainty in the $K_{I}$ estimation reveals the same trends as in the pure X-DIC case. However, the values are lower by a factor of about 4 . From these results, one may think that with the choice of $\lambda=0.25$, which is optimal for the displacement jump uncertainty, the displacement field is still affected by noise. As shown by Figure 13a, the influence of this remaining noise is reduced by adopting larger integration domains. For $\lambda=0.0001$ the uncertainty appears to be independent of the size of the integration domain because of the strong reduction of the noise level affecting the displacement field obtained with such a strong penalization of the momentum and constitutive equations. As an alternative for reducing the noise influence on the stress intensity factors estimation, the interested reader may refer to (Réthoré et al., 2006) for an optimization of the virtual crack extension field to lower the sensitivity to measurement uncertainties.

The results for $K_{I}$ are presented in Figure 13b. Even though the interaction integral is theoretically domain-independent, the mode $I$ stress intensity factor from the analysis of a pure correlation result remains oscillating. As the integrated approach restores the independence conditions of the interaction integral (balance of momentum, traction free crack faces), $K_{I}$ computed from the XI-DIC displacement field reveals 


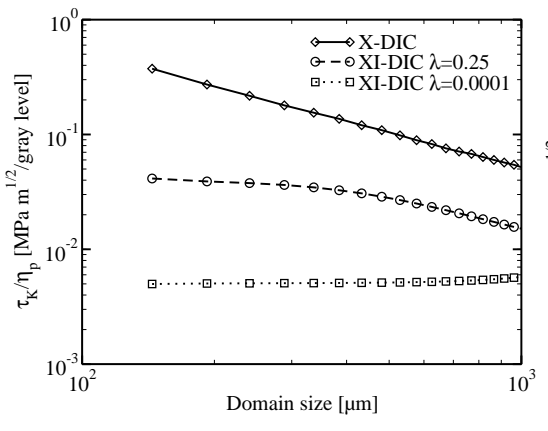

$-a-$

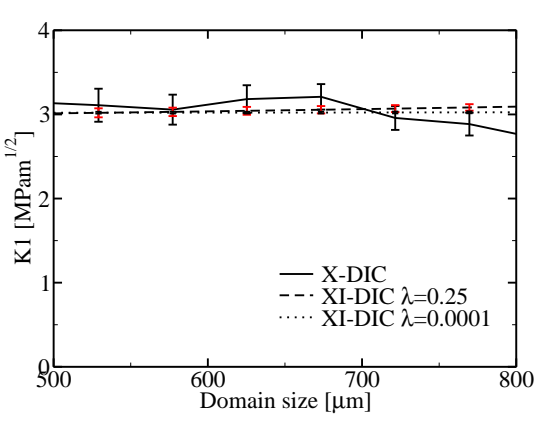

$-\mathrm{b}-$

Figure 13. Uncertainty (a) and mode I stress intensity factor (b) obtained from the $X$-DIC and XI-DIC results.

only tiny variations with respect to domain size. Adopting a lower coupling parameter meaning a stronger mechanical filtering, the domain independence is better achieved with lower values of $\lambda$. The error bars depicted in Figure 13 correspond to an image noise of 2 gray levels. With a domain size of $580 \mu \mathrm{m}$, a value of $3.06 \pm 0.18 \mathrm{MPa} \sqrt{\mathrm{m}}$ is obtained for X-DIC whereas the XI-DIC results are $3.03 \pm 0.05 \mathrm{MPa} \sqrt{\mathrm{m}}$ for $\lambda=0.25$ and $3.02 \pm 0.01 \mathrm{MPa} \sqrt{\mathrm{m}}$ for $\lambda=0.0001$. Those three results are consistent with respect to the uncertainty ranges obtained for each case. Let us also note that the XI-DIC results with the initial crack tip position are in good agreement with the results of the integrated approach proposed by Roux et al. (2006). The much lower uncertainty level obtained with the XI-DIC approach are to be put into perspective by a higher sensitivity to the crack tip location.

\subsection{Location sensitivity}

From the previous section, the noise sensitivity of the identification procedure of the stress intensity factors is obtained. Another important feature is the crack tip location and one may wonder about the sensitivity of the stress intensity factors estimation to a bias on the location of the crack tip. For this purpose, Figure 14 shows the change of $K_{I}$ when the crack tip is translated from its initial position. A linear regression enables us to estimate the sensitivity of the $K_{I}$ measurement chain to $0.012 \mathrm{MPa} \sqrt{\mathrm{m}} /$ pixel for the XI-DIC algorithm whereas the uncertainty due to the image noise remains higher than the location sensitivity for the X-DIC technique.

Yet, the three techniques used herein to determine the position of the crack tip give very consistent results that increase the confidence in a 43-pixel shift for the present case. Furthermore, using the super-singular component of the displacement field to locate the position of the crack tip, the following system is solved

$$
\mathbf{L V}=\mathbf{B}
$$




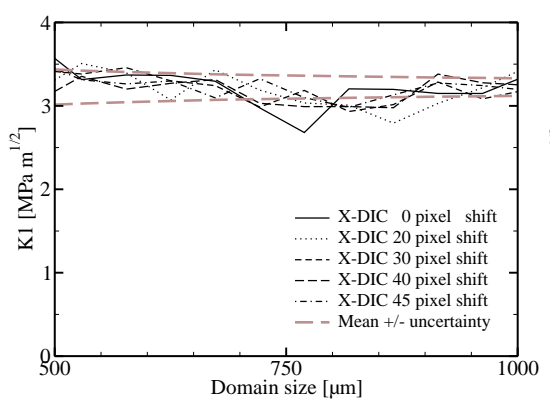

$-\mathrm{a}-$

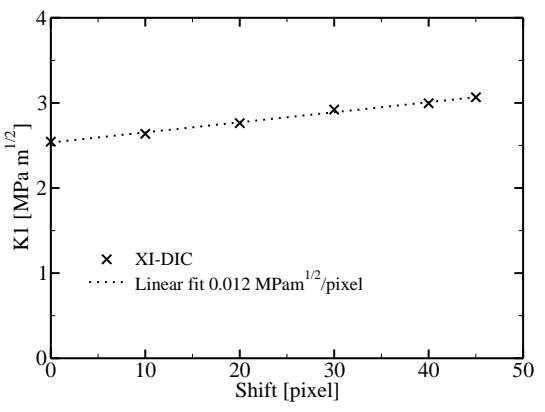

$-\mathrm{b}-$

Figure 14. Location sensitivity: for the X-DIC displacement field (a) the uncertainty level due to image noise is higher than the influence of a mismatch of the crack tip position, $K_{I}$ as a function of the crack tip shift for XI-DIC (b).

where $\mathbf{V}$ contains the amplitudes $a_{n}^{j}$ of the displacement field corresponding to the Kolossov-Muskhelishvili integer-labeled families of solutions and $\mathbf{L}, \mathbf{B}$ have the following expressions

$$
L_{i j}=\iint \Phi_{i} \Phi_{j} d S \quad B_{j}=\iint \Phi_{j} \mathbf{U} d S
$$

For a homogeneous perturbation $\eta_{u}$ of the displacement field a zero-mean perturbation $\delta V$ of the fitted amplitudes is obtained. The variance $\left\langle\delta V^{2}\right\rangle$ of this perturbation reads

$$
\left\langle\delta V^{2}\right\rangle=\mathbf{L}^{-1} \mathbf{L} \mathbf{L}^{-1}\left\langle\eta_{u}^{2}\right\rangle=\mathbf{L}^{-1}\left\langle\eta_{u}^{2}\right\rangle
$$

Then the variance of the offset distance of the crack tip is evaluated in the vicinity of $a_{-1}^{1} \approx 0$ as

$$
\left\langle\delta x_{1}^{2}\right\rangle=\left(\frac{2}{a_{1}^{1}}\right)^{2} \mathbf{L}_{o o}^{-1}\left\langle\eta_{u}^{2}\right\rangle
$$

where $o$ is the index corresponding to $\Phi_{-1}^{1}$. Accounting for a value $\left\langle\eta_{u}^{2}\right\rangle$ of about $6 \times$ $10^{-4} \mathrm{pixel}^{2}$, the uncertainty in the crack tip offset is \pm 0.5 pixel. Because a description of the crack geometry at the pixel-scale is adopted, the positioning error is set to \pm 1 pixel. Consequently, the crack tip location is accurate and the uncertainty for $K_{I}$ due to the positioning uncertainty is as low as $\pm 0.012 \mathrm{MPa} \sqrt{\mathrm{m}}$. Adding the error from the interaction integral, the global uncertainty for $K_{I}$ using the XI-DIC strategy is $\pm 0.022 \mathrm{MPa} \sqrt{\mathrm{m}}$ for $\lambda=0.0001$. This error remains at least 5 times less than the uncertainty of the X-DIC identification procedure. All these results show that the value $\lambda=0.0001$ is a good estimate and can be used for the XI-DIC procedure. Consequently, the computation time required for XI-DIC compared with X-DIC is comparable, the main difference is given by the assembly of a stiffness matrix whose elementary terms are easily known for Q4 elements. 


\section{Summary}

Considering static admissibility conditions in the determination of the displacement field by digital image correlation, a mechanical filter is obtained. Using the sum of two objective functions, one based on the residuals associated with the correlation of reference and deformed images and a second associated with the internal forces (in a finite element framework), the correlation algorithm is penalized by a minimization of the equilibrium gap condition. The resulting fields are closer to the space of admissible elastic solutions of the problem (and statically admissible in one numerically accessible limit). The proposed approach enables for a more robust analysis than using a pure correlation algorithm. In the application discussed herein of crack detection in a ceramic specimen, the displacement field is sub-pixel and the displacement jump over a 15 pixel radius circle remains in the uncertainty range (i.e., about 0.025 pixel). The detection of the actual position of the crack tip is proposed based on a criterion exploiting supersingular crack fields. The final estimate of the crack tip position is estimated to be accurate to ca. 1 pixel (or less than $2.0 \mu \mathrm{m}$ ).

After an appropriate position is assigned to the crack tip, stress intensity factors are estimated using an interaction integral. The domain independence is not obtained from pure correlation results. Conversely, the integrated approach restores the conditions to achieve the stability of the interaction integral. However, the results from the two approaches are in good agreement to the uncertainty of the X-DIC estimation of the displacement field. Using such an integrated approach, the uncertainty of the stress intensity factors estimate reaches the very low value of about $1 \%$.

In the present paper, the coupling parameter is considered as a constant over the entire region of interest. For many reasons, for example if the process zone is extensively developed around the crack tip and the assumption of a linear elastic material is not valid over this region, the coupling parameter may be considered as a function of space. A pure correlation problem may subsequently be solved over the region wherein the assumed material behavior failed at modeling the actual one. The use of a spatially variable coupling parameter and the use of such an integrated digital image correlation technique for the identification of non-linear material behavior will be the purpose of further studies.

\section{Acknowledgements}

This work was funded by the CETIM Foundation grant entitled PROPAVANFIS: "Advanced methods for the experimental and numerical analyses of crack propagations under complex loadings." 


\section{Bibliographie}

Anbanto-Bueno J., Lambros J., "Investigation of crack growth in functionally graded materials using digital image correlation”, Engineering Fracture Mechanics, vol. 69, p. 1695-1711, 2002.

Besnard G., Hild F., Roux S., "'Finite-element' displacement fields analysis from digital images: Application to Portevin-Le Châtelier bands", Experimental Mechanics, vol. 46, no. 6, p. 789-803, 2006.

Claire D., Hild F., Roux S., "A finite element formulation to identify damage fields: The equilibrium gap method", International Journal for Numerical Methods in Engineering, vol. 61, p. 189-208, 2004.

Forquin P., Rota L., Charles Y., Hild F., "A method to determine the toughness scatter of brittle materials", International Journal of Fracture, vol. 125, no. 1, p. 171-187, 2004.

Hild F., Roux S., "Digital image correlation: from displacement measurement to identification of elastic properties-A review", Strain, vol. 42, p. 69-80, 2006.

Irwin G., "Analysis of the stresses and strains near the end of a crack traversing a plate", ASME Journal Applied Mechanics, vol. 24, p. 361-364, 1957.

McNeill S., Peters W., Sutton M., "Estimation of stress intensity factor by digital image correlation”, Engineering Fracture Mechanics, vol. 28, no. 1, p. 101-112, 1987.

Moës N., Dolbow J., Belytschko T., "A finite element method for crack growth without remeshing”, International Journal for Numerical Methods in Engineering, vol. 46, no. 1, p. 133150, 1999.

Réthoré J., Gravouil A., Morestin F., Combescure A., "Estimation of mixed-mode stress intensity factors using digital image correlation and an interaction integral", International Journal of Fracture, vol. 132, no. 1, p. 65-79, 2005.

Réthoré J., Hild F., Roux S., "Extended digital image correlation with crack shape optimization", International Journal for Numerical Methods in Engineering, http://dx.doi.org/10.1002/nme.2070, 2007.

Réthoré J., Roux S., Hild F., "Noise-robust Stress Intensity Factor Determination from Kinematic Field Measurements", Engineering Fracture Mechanics, http://www.sciencedirect.com/science/journal/00137944, 2007.

Roux S., Hild F., "Stress intensity factor measurement from digital image correlation: postprocessing and integrated approaches", International Journal of Fracture, vol. 140, no. 1-4, p. 141-157, 2006.

Sutton M., McNeill S., Helm J., Chao Y., Advances in two-dimensional and three-dimensional computer vision, in "Photomechanics", Springer, p. 323-372, 2000.

Williams M., "On the stress distribution at the base of a stationary crack", ASME Journal Applied Mechanics, vol. 24, p. 109-114, 1957.

Zi G., Belytschko T., "New crack-tip elements for XFEM and applications to cohesive cracks", International Journal for Numerical Methods in Engineering, vol. 57, no. 15, p. 2221-2240, 2003. 